TRANSACTIONS OF THE

AMERICAN MATHEMATICAL SOCIETY

Volume 360, Number 10, October 2008, Pages 5247-5264

S 0002-9947(08)04556-X

Article electronically published on April 10, 2008

\title{
CONFORMAL COCHAINS
}

\author{
SCOTT O. WILSON
}

\begin{abstract}
In this paper we define holomorphic cochains and an associated period matrix for triangulated closed topological surfaces. We use the combinatorial Hodge star operator introduced in the author's paper of 2007, which depends on the choice of an inner product on the simplicial 1-cochains.

We prove that for a triangulated Riemannian 2-manifold (or a Riemann surface), and a particularly nice choice of inner product, the combinatorial period matrix converges to the (conformal) Riemann period matrix as the mesh of the triangulation tends to zero.
\end{abstract}

\section{INTRODUCTION}

In this paper we use the combinatorial Hodge star operator, introduced in [18, to define holomorphic cochains of a triangulated (topological) surface whose simplicial 1-cochains are equipped with a positive definite inner product. We study the periods of these cochains and prove they satisfy the bi-linear relations of Riemann. We also defined the associated combinatorial period matrix.

Using the inner product introduced in [1], we prove the following result:

Theorem 1.1. The combinatorial period matrix of a triangulated Riemmanian 2-manifold converges to the conformal period matrix of the associated Riemann surface, as the mesh of the triangulation tends to zero.

By Torelli's theorem, this shows that the conformal structure of a Riemann surface can be computed to any desired level of accuracy, from finite algebraic data. It also suggests a link between statistical mechanics and conformal field theory, where it is known that the partition function may be expressed in terms of theta functions of the conformal period matrix [7]; see also [8], 10]. This link is consistent with our philosophy that cochains provide a precise link between finiteelement/lattice theories and smooth differential geometry.

The above convergence statements are made precise by using an embedding of simplicial cochains into differential forms, first introduced by Whitney [16]. This approach was used quite successfully by Dodziuk [1, and later by Dodziuk and Patodi [2], to show that cochains provide a good approximation to smooth differential forms, and that the combinatorial Laplacian converges to the smooth Laplacian. This formalism will be reviewed in section 3 .

In section 4 we describe a cochain cup product that will be used in section 5 to define the combinatorial star operator. Also in section 5, we review some of the

Received by the editors August 8, 2006.

2000 Mathematics Subject Classification. Primary 57R57, 32G20; Secondary 30F99.

Key words and phrases. Cochains, Hodge-star, Riemann surface, period matrices.

(C)2008 American Mathematical Society

Reverts to public domain 28 years from publication 
properties of the combinatorial star operator and some results on its convergence to the smooth Hodge star operator.

In section [6 we use the combinatorial star operator for surfaces to introduce combinatorial period matrices. In the process, we define holomorphic and antiholomorphic cochains, prove some (Riemann) bi-linear relations that the periods satisfy, and prove our convergence result, mentioned above.

\section{BACKGROUND AND ACKNOWLEDGMENTS}

In connection with our application of the combinatorial star operator to surfaces, in particular proving the convergence of our combinatorial period matrix to the conformal period matrix, Mercat has a related result in [10]. As part of his extensive study of what he calls "discrete Riemann surfaces", he assigns to any such object a "period matrix" of twice the expected size. He shows that there are two submatrices of the appropriate dimension $(g \times g)$ satisfying the property that, given what he calls "a refining sequence of critical maps," they both converge to the continuum period matrix of an associated Riemann surface. This uses his results on discrete holomorphy approximations presented in 11. While our approach differs greatly, e.g there is no "doubling" of complexes or operators, the results seem similar in spirit.

There is a related application of period matrices presented in $[6$. There Xianfeng $\mathrm{Gu}$ and Shing-Tung Yau give algorithms for computing a conformal structure of a surface, though no convergence statements are formulated or proven. They point out that these algorithms can be implemented on the simplicial cochains, and have generated some impressive images (of human faces!) from them.

The author thanks Dennis Sullivan for his many useful comments and suggestions on this work, Jozef Dodziuk for his help with several points in his papers [1, 2, and Ruben Costa-Santos and Barry McCoy for their inspiring work in 8]. Also, the author thanks Anthony Phillips, Lowell Jones, Jim Stasheff and Robert Kotiuga and the referee for comments and corrections on earlier versions.

\section{WHITNEY FORMS}

In this section, we review some definitions and results on Whitney forms. These ideas, originating with Whitney [16, and made more precise by the work of Dodziuk [1], use a linear embedding of simplicial cochains into piecewise-linear differential forms to show that cochains provide a good approximation of differential forms. The techniques involved illustrate a tight (and analytically precise) connection between cochains and forms, and will be used later to give precise meaning to our constructions of period matrices. In particular, our convergence statements about combinatorial and smooth objects will be cast in a way similar to the results reviewed in this section.

Let $M$ be a closed smooth $n$-manifold and $K$ a fixed $C^{\infty}$ triangulation of $M$. We identify $|K|$ and $M$ and fix an ordering of the vertices of $K$. Let $C^{j}$ denote the simplicial cochains of degree $j$ of $K$ with values in $\mathbb{R}$. Given the ordering of the vertices of $K$, we have a coboundary operator $\delta: C^{j} \rightarrow C^{j+1}$. Let $\mu_{i}$ denote the barycentric coordinate corresponding to the $i^{\text {th }}$ vertex $p_{i}$ of $K$. Since $M$ is compact, we may identify the cochains and chains of $K$ and for $c \in C^{j}$ write $c=\sum_{\tau} c_{\tau} \cdot \tau$ where $c_{\tau} \in \mathbb{R}$ and is the sum over all $j$-simplices $\tau$ of $K$. We write $\tau=\left[p_{0}, p_{1}, \ldots, p_{j}\right]$ of $K$ with the vertices in an increasing sequence with respect to 
the ordering of vertices in $K$. We now define the Whitney embedding of cochains into piecewise-linear differential forms.

Definition 3.1. For $\tau$ as above, we define

$$
W \tau=j ! \sum_{i=0}^{j}(-1)^{i} \mu_{i} d \mu_{0} \wedge \cdots \wedge \widehat{d \mu_{i}} \wedge \cdots \wedge d \mu_{j} .
$$

$W$ is defined on all of $C^{j}$ by extending linearly.

Note that the coordinates $\mu_{\alpha}$ are not even of class $C^{1}$, but they are $C^{\infty}$ on the interior of any $n$-simplex of $K$. Hence, $d \mu_{\alpha}$ is defined and $W \tau$ is well defined. By the same consideration, $d W$ is also well defined, where $d$ denotes exterior derivative. Note both sides of the definition of $W$ are alternating, so this map is well defined for all simplices regardless of the ordering of vertices.

Several properties of the map $W$ are given below. See [16, [1], 2] for details.

Proposition 3.2. The following hold:

(1) $W \tau=0$ on $M \backslash \overline{S t(\tau)}$,

(2) $d W=W \delta$,

where St denotes the open star and - denotes closure.

One also has a map $R$ from differential forms to $C^{j}(K)$, the de Rham map, given by integration. Precisely, for any differential form $\omega$ and chain $c$ we have:

$$
R \omega(c)=\int_{c} \omega .
$$

It is a theorem of de Rham that this map is a quasi-isomorphism (it is a chain map by Stokes's Theorem). There are many classes of differential forms on which $R$ is defined $\left(C^{1}\right.$, smooth, piecewise, etc.). It is certainly defined on the image of the Whitney map $W$, and one can check that $R W=I d$; see [16, [1], 2].

Before stating Dodziuk and Patodi's theorem, that $W R$ is approximately equal to the identity, we first give some definitions concerning triangulations. They also appear in [2].

Definition 3.3. Let $K$ be a triangulation of an $n$-dimensional manifold $M$. The mesh $\eta=\eta(K)$ of a triangulation is

$$
\eta=\sup r(p, q),
$$

where $r$ means the geodesic distance in $M$ and the supremum is taken over all pairs of vertices $p, q$ of a 1 -simplex in $K$.

The fullness $\Theta=\Theta(K)$ of a triangulation $K$ is

$$
\Theta(K)=\inf \frac{\operatorname{vol}(\sigma)}{\eta^{n}}
$$

where the inf is taken over all $n$-simplexes $\sigma$ of $K$ and $\operatorname{vol}(\sigma)$ is the Riemannian volume of $\sigma$, as a Riemannian submanifold of $M$.

A Euclidean analogue of the following lemma was proven by Whitney in [16] (IV.14). 
Lemma 3.4. Let $M$ be a smooth Riemannian n-manifold.

(1) Let $K$ be a smooth triangulation of $M$. Then there is a positive constant $\Theta_{0}>0$ and a sequence of subdivisions $K_{1}, K_{2}, \ldots$ of $K$ such that $\lim _{n \rightarrow \infty} \eta\left(K_{n}\right)=0$ and $\Theta\left(K_{n}\right) \geq \Theta_{0}$ for all $n$.

(2) Let $\Theta_{0}>0$. There exist positive constants $C_{1}, C_{2}$ depending on $M$ and $\Theta_{0}$ such that for all smooth triangulations $K$ of $M$ satisfying $\Theta(K) \geq \Theta_{0}$, all $n$-simplexes of $\sigma=\left[p_{0}, p_{1}, \ldots, p_{n}\right]$ and vertices $p_{k}$ of $\sigma$,

$$
\begin{aligned}
\operatorname{vol}(\sigma) & \leq C_{1} \cdot \eta^{n}, \\
C_{2} \cdot \eta & \leq r\left(p_{k}, \sigma_{p_{k}}\right),
\end{aligned}
$$

where $r$ is the Riemannian distance, $\operatorname{vol}(\sigma)$ is the Riemannian volume, and $\sigma_{p_{k}}=\left[p_{0}, \ldots, p_{k-1}, p_{k+1}, \ldots, p_{n}\right]$ is the face of $\sigma$ opposite to $p_{k}$.

Since any two metrics on $M$ are commensurable, the lemma follows from Whitney's Euclidean result; see also [2].

We consider only those triangulations with fullness bounded below by some positive real constant $\Theta_{0}$. By the lemma, this guarantees that the volume of a simplex is on the order of its mesh raised to the power of its dimension. Geometrically, this means that in a sequence of triangulations, the shapes do not become too thin. (In fact, Whitney's standard subdivisions yield only finitely many shapes, and can be used to prove the first part of the lemma.) Most of our estimates depend on $\Theta_{0}$, as can be seen in the proofs. We'll not indicate this dependence in the statements.

The following theorems are proved by Dodziuk and Patodi in [2]. They show that for a fine triangulation, $W R$ is approximately equal to the identity. In this sense, the theorems give precise meaning to the statement: for a fine triangulation, cochains provide a good approximation to differential forms.

Theorem 3.5. Let $\omega$ be a smooth form on $M$, and $\sigma$ be an $n$-simplex of $K$. There exists a constant $C$, independent of $\omega, K$ and $\sigma$, such that

$$
|\omega-W R \omega|_{p} \leq C \cdot \sup \left|\frac{\partial \omega}{\partial x^{i}}\right| \cdot \eta
$$

for all $p \in \sigma$. The supremum is taken over all $p \in \sigma$ and $i=1,2, \ldots, n$, and the partial derivatives are taken with respect to a coordinate neighborhood containing $\sigma$.

By integrating the above point-wise and applying a Sobolev inequality, Dodziuk and Patodi 2 obtain the following

Corollary 3.6. There exist a positive constant $C$ and a positive integer $m$, independent of $K$, such that

$$
\|\omega-W R \omega\| \leq C \cdot\left\|(I d+\Delta)^{m} \omega\right\| \cdot \eta
$$

for all $C^{\infty} j$-forms $\omega$ on $M$.

Now suppose the cochains $C(K)$ are equipped with a positive definite inner product $\langle$,$\rangle such that, for distinct i, j, C^{i}(K)$ and $C^{j}(K)$ are orthogonal. Then one can define further structures on the cochains. In particular, we have the following

Definition 3.7. The adjoint of $\delta$, denoted by $\delta^{*}$, is defined by $\left\langle\delta^{*} \sigma, \tau\right\rangle=\langle\sigma, \delta \tau\rangle$. 
Note that $\delta^{*}: C^{j}(K) \rightarrow C^{j-1}(K)$ is also squares to zero. One can also define

Definition 3.8. The combinatorial Laplacian is defined to be $\boldsymbol{\Lambda}=\delta^{*} \delta+\delta \delta^{*}$.

Clearly, both $\delta^{*}$ and $\boldsymbol{\Delta}$ depend upon the choice of inner product. For any choice of non-degenerate inner product, these operators give rise to a combinatorial Hodge theory: the space of harmonic $j$-cochains of $K$ is defined to be

$$
\mathcal{H} C^{j}(K)=\left\{a \in C^{j} \mid \mathbf{\Delta} a=\delta a=\delta^{*} a=0\right\} .
$$

The following theorem is due to Eckmann [4]:

Theorem 3.9. Let $(C, \delta)$ be a finite dimensional complex with inner product $\langle$,$\rangle ,$ and induced adjoint $\delta^{*}$ as above. There is an orthogonal direct sum decomposition

$$
C^{j}(K) \cong \delta C^{j-1}(K) \oplus \mathcal{H} C^{j}(K) \oplus \delta^{*} C^{j+1}(K)
$$

and $\mathcal{H} C^{j}(K) \cong H^{j}(K)$, the cohomology of $(K, \delta)$ in degree $j$.

If $K$ is a triangulation of a Riemannian manifold $M$, then there is a particularly nice inner product on $C(K)$, which we'll call the Whitney inner product. It is induced by the metric $\langle$,$\rangle on differential forms (which, in turn, is induced by the$ Riemannian metric) and the Whitney embedding of cochains into piecewise-linear forms. We'll use the same notation $\langle$,$\rangle for this pairing on C:\langle\sigma, \tau\rangle=\langle W \sigma, W \tau\rangle$.

It is proven in [1] that the Whitney inner product on $C$ is positive definite. Further consideration of this inner product will be given in later sections. For now, following [1] and 2, we describe how the combinatorial Hodge theory, induced by the Whitney inner product, is related to the smooth Hodge theory. Precisely, we have the following theorem due to Dodziuk and Patodi [2, which shows that the approximation $W R \approx I d$ respects the Hodge decompositions of $\Omega(M)$ and $C(K)$.

Theorem 3.10. Let $\omega \in \Omega^{j}(M)$ and $R \omega \in C^{j}(K)$ have Hodge decompositions

$$
\begin{aligned}
\omega & =d \omega_{1}+\omega_{2}+d^{*} \omega_{3}, \\
R \omega & =\delta a_{1}+a_{2}+\delta^{*} a_{3} .
\end{aligned}
$$

Then,

$$
\begin{aligned}
\left\|d \omega_{1}-W \delta a_{1}\right\| & \leq \lambda \cdot\left\|(I d+\Delta)^{m} \omega\right\| \cdot \eta, \\
\left\|\omega_{2}-W a_{2}\right\| & \leq \lambda \cdot\left\|(I d+\Delta)^{m} \omega\right\| \cdot \eta, \\
\left\|d^{*} \omega_{3}-W \delta^{*} a_{3}\right\| & \leq \lambda \cdot\left\|(I d+\Delta)^{m} \omega\right\| \cdot \eta
\end{aligned}
$$

where $\lambda$ and $m$ are independent of $\omega$ and $K$.

\section{Cochain product}

In this section we describe a commutative, but non-associative, cochain product. It will be used to define the combinatorial star operator.

Definition 4.1. We define $\cup: C^{j}(K) \otimes C^{k}(K) \rightarrow C^{j+k}(K)$ by

$$
\sigma \cup \tau=R(W \sigma \wedge W \tau) .
$$

Since $R$ and $W$ are chain maps with respect to $d$ and $\delta$, it follows that $\delta$ is a derivation of $\cup$, that is, $\delta(\sigma \cup \tau)=\delta \sigma \cup \tau+(-1)^{\operatorname{deg}(\sigma)} \sigma \cup \delta \tau$. Also, since $\wedge$ is graded commutative, $\cup$ is as well: $\sigma \cup \tau=(-1)^{\operatorname{deg}(\tau) \operatorname{deg}(\sigma)} \tau \cup \sigma$. It follows from a theorem of Whitney [17 that the product $\cup$ induces the same map on cohomology as the usual (Alexander-Whitney) simplicial cochain product. 


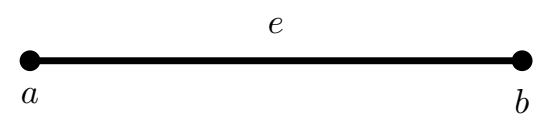

FiguRE 1. Cochain product on the unit interval

We now state a combinatorial description of this cup product; this also appears in [3. An easy way to state this is as follows: the product of a $j$-simplex and $k$-simplex is zero unless these simplices span a common $(j+k)$-simplex, in which case the product is a rational multiple of this $(j+k)$-simplex.

Proposition 4.2. Let $\sigma=\left[p_{\alpha_{0}}, p_{\alpha_{1}}, \ldots, p_{\alpha_{j}}\right] \in C^{j}(K)$ and $\tau=\left[p_{\beta_{0}}, p_{\beta_{1}}, \ldots, p_{\beta_{k}}\right] \in$ $C^{k}(K)$. Then $\sigma \cup \tau$ is zero unless $\sigma$ and $\tau$ intersect in exactly one vertex and span $a(j+k)$-simplex $v$, in which case, for $\tau=\left[p_{\alpha_{j}}, p_{\alpha_{j+1}}, \ldots, p_{\alpha_{j+k}}\right]$, we have:

$$
\begin{aligned}
\sigma \cup \tau & =\left[p_{\alpha_{0}}, p_{\alpha_{1}}, \ldots, p_{\alpha_{j}}\right] \cup\left[p_{\alpha_{j}}, p_{\alpha_{j+1}}, \ldots, p_{\alpha_{j+k}}\right] \\
& =\epsilon(\sigma, \tau) \frac{j ! k !}{(j+k+1) !}\left[p_{\alpha_{0}}, p_{\alpha_{1}}, \ldots, p_{\alpha_{j+k}}\right],
\end{aligned}
$$

where $\epsilon(\sigma, \tau)$ is determined by:

$$
\operatorname{orientation}(\sigma) \cdot \operatorname{orientation}(\tau)=\epsilon(\sigma, \tau) \cdot \operatorname{orientation}(v) .
$$

Proof. See [3] or later [18] for the calculation.

A special case of this result was derived by Ranicki and Sullivan 12 for $K$ a triangulation of a $4 k$-manifold and $\sigma, \tau$ of complimentary dimension. In that paper, they showed that the pairing given by $\cup$ restricted to simplices of complimentary dimension gives rise to a semi-local combinatorial formula for the signature of a $4 k$-manifold.

Remark 4.3. The constant 0 -cochain which evaluates to 1 on all 0 -simplices is the unit of the differential graded commutative (but non-associative) algebra $\left(C^{*}, \delta, \cup\right)$.

Example 4.4. The product $\cup$ is not associative. For example, in Figure 1 ( $a \cup$ b) $\cup e=0$, since $a$ and $b$ do not span a 0 -simplex, but $a \cup(b \cup e)=\frac{1}{4} e$.

Remark 4.5. In 18 it is shown that this cup product converges (in a precise sense described there) to the wedge product on differential forms. In light of Remark 4.3. those results generalize Theorem 3.5 and Corollary 3.6, but will not be needed here.

\section{Combinatorial Star Operator}

In this section we recall the definition of the combinatorial star operator $\star$ introduced in [18] as well as a theorem showing it provides a good approximation to the smooth Hodge-star. Let $K$ be a triangulation of a closed oriented manifold $M$, with simplicial cochains $C=\bigoplus_{j} C^{j}$.

Definition 5.1. Let $\langle$,$\rangle be a positive definite inner product on C$ such that $C^{i}$ is orthogonal to $C^{j}$ for $i \neq j$. For $\sigma \in C^{j}$ we define $\star \sigma \in C^{n-j}$ by:

$$
\langle\star \sigma, \tau\rangle=(\sigma \cup \tau)[M]
$$

where $[M]$ denotes the fundamental class of $M$. 
We emphasize that, the essential ingredients of a star operator are Poincaré Duality and an inner product 1 We can regard the inner product as giving some geometric structure to the space. In particular it gives lengths of edges and angles between them. As in the smooth setting, the star operator depends on the choice of inner product (or Riemannian metric).

Here are some elementary properties of $\star$.

Lemma 5.2. The following hold:

(1) $\star \delta=(-1)^{j+1} \delta^{*} \star$, i.e. $\star$ is a chain map.

(2) For $\sigma \in C^{j}$ and $\tau \in C^{n-j},\langle\star \sigma, \tau\rangle=(-1)^{j(n-j)}\langle\sigma, \star \tau\rangle$, i.e. $\star$ is (graded) skew-adjoint.

(3) $\star$ induces isomorphisms $\mathcal{H} C^{j}(K) \rightarrow \mathcal{H} C^{n-j}(K)$ on harmonic cochains.

Proof. The first two proofs are computational: the first follows since $\delta$ is a graded derivation of $U$ and the second follows since $U$ is graded commutative; see [18. For the last assertion, the induced map can be described by the composition of inclusion, $\star$, and orthogonal projection onto harmonics:

$$
\mathcal{H} C^{j}(K) \hookrightarrow C^{j}(K) \rightarrow C^{n-j}(K) \rightarrow \mathcal{H} C^{n-j}(K)
$$

Equivalently, this composition can be expressed by the operator (also denoted $\star$ ) given by

$$
\langle\star \sigma, \tau\rangle=(\sigma \cup \tau)[M]
$$

where now $\sigma \in \mathcal{H} C^{j}(K)$ and $\tau \in \mathcal{H} C^{n-j}(K)$. The harmonic cochains give cocycle representatives for the cohomology of $C(K)$. By Poincaré Duality the pairing on cohomology, and therefore also on harmonics, is non-degenerate. The inner product is also non-degenerate, so the result follows.

We remark here that $\star: C \rightarrow C$ is in general not invertible, since the cochain cup product may not give rise to a non-degenerate pairing on the cochain level 2 Thus, $\star$ is in general not an orthogonal map, and $\star^{2} \neq \pm$ Id. Lastly, $\delta^{*} \star \neq \star \delta$.

For the remainder of this section, we'll fix the inner product on $C$ to be the Whitney inner product, so that $\star$ is the star operator induced by the Whitney inner product. This will be essential in showing that $\star$ converges to the smooth Hodge star $\star$, which is defined using the Riemannian metric. First, a useful lemma. Let $\pi$ denote the orthogonal projection of piecewise-linear forms onto the image of $C^{j}(K)$ under the Whitney embedding $W$.

Lemma 5.3. $W \star=\pi \star W$.

Proof. Let $a \in C^{j}(K)$ and $b \in C^{n-j}(K)$. Note that $\star W a$ is, in general, not a Whitney form. We compute:

$$
\langle W \star a, W b\rangle=\langle\star a, b\rangle=\int_{M} W a \wedge W b=\langle\star W a, W b\rangle .
$$

Thus, $W \star a$ and $\star W a$ have the same inner product with all forms in the image of $W$, so $W \star=\pi \star W$.

\footnotetext{
${ }^{1}$ The referee kindly pointed out that, unlike the Hodge star, the combinatorial star operator does not require the star operator on cochains of all degrees to be induced from the star operator on 1-cochains, and this may be useful in applications.

${ }^{2} \mathrm{~A}$ geometric description of the kernel in terms of the combinatorics of the triangulation would be interesting. The author does not understand this, or even conditions under which a cochain cup product gives rise to a non-degenerate pairing.
} 
We now recall the convergence theorem of $\star[18]$.

Theorem 5.4. Let $M$ be a Riemannian manifold with triangulation $K$ of mesh $\eta$. There exist a positive constant $C$ and a positive integer $m$, independent of $K$, such that

$$
\|\star \omega-W \star R \omega\| \leq C \cdot\left\|(I d+\Delta)^{m} \omega\right\| \cdot \eta
$$

for all $C^{\infty}$ differential forms $\omega$ on $M$.

Proof. Since the proof is short, we'll give it here using Theorem 3.5:

$$
\begin{aligned}
\|\star \omega-W \star R \omega\| & =\|\star \omega-\pi \star W R \omega\| \\
& \leq\|\star \omega-\star W R \omega\|+\|\star W R \omega-\pi \star W R \omega\| \\
& \leq\|\star\|\|\omega-W R \omega\|+\|\star W R \omega-W R \star \omega\| \\
& \leq\|\omega-W R \omega\|+\|\star W R \omega-\star \omega\|+\|\star \omega-W R \star \omega\| \\
& \leq 2\|\omega-W R \omega\|+\|\star \omega-W R \star \omega\| \\
& \leq 3 C \cdot\left\|(I d+\Delta)^{m} \omega\right\| \cdot \eta .
\end{aligned}
$$

Crucially, the operator $\star$ also respects the Hodge decompositions of $C(K)$ and smooth forms $\Omega(M)$ in the following sense:

Theorem 5.5. Let $M$ be a Riemannian manifold with triangulation $K$ of mesh $\eta$. Let $\omega \in \Omega^{j}(M)$ and $R \omega \in C^{j}(K)$ have Hodge decompositions

$$
\begin{aligned}
\omega & =d \omega_{1}+\omega_{2}+d^{*} \omega_{3}, \\
R \omega & =\delta a_{1}+a_{2}+\delta^{*} a_{3} .
\end{aligned}
$$

There exist a positive constant $C$ and a positive integer $m$, independent of $K$, such that

$$
\begin{aligned}
\left\|\star d \omega_{1}-W \star \delta a_{1}\right\| & \leq C \cdot\left(\left\|(I d+\Delta)^{m} \omega\right\|+\left\|(I d+\Delta)^{m} d \omega_{1}\right\|\right) \cdot \eta, \\
\left\|\star \omega_{2}-W \star a_{2}\right\| & \leq C \cdot\left(\left\|(I d+\Delta)^{m} \omega\right\|+\left\|(I d+\Delta)^{m} \omega_{2}\right\|\right) \cdot \eta, \\
\left\|\star d^{*} \omega_{3}-W \star \delta^{*} a_{3}\right\| & \leq C \cdot\left(\left\|(I d+\Delta)^{m} \omega\right\|+\left\|(I d+\Delta)^{m} d^{*} \omega_{3}\right\|\right) \cdot \eta .
\end{aligned}
$$

Proof. A proof of this slightly more complicated estimate appears in [18].

\section{Surfaces AND Period matrices}

In this section we develop the main results of this paper involving the combinatorial star operator on a triangulated closed surface. As motivation, let us recall some facts from the analytic setting.

Let $M$ be a Riemann surface. The Hodge-star operator on the complex valued 1-forms of $M$ may be defined in local coordinates by $\star d x=d y$ and $\star d y=-d x$ and extended over $\mathbb{C}$ linearly. One can check that this is well defined using the CauchyRiemann equations for the coordinate interchanges. The Hodge-star operator restricts to an orthogonal automorphism of complex valued 1-forms that squares to -Id. Furthermore, the harmonic 1-forms split into an orthogonal sum of holomorphic and anti-holomorphic 1-forms corresponding to the $-i$ and $+i$ eigenspaces of the Hodge-star operator.

In Riemann's study of periods on surfaces, i.e. the integrals of holomorphic and anti-holomorphic 1-forms over cycles, he showed that for any fixed homology basis these periods satisfy the so-called bi-linear relations. Furthermore, choosing 
a particular basis for the holomorphic 1-forms gives rise to a period matrix, which, by Torelli's theorem, determines the conformal structure of the Riemann surface. These period matrices lie in what is called the Siegel upper half space. (Two references for this material are [15] and [5].) An unsolved problem, called the Schottky problem, is to determine which points in the Siegel upper half space represent the period matrix of a Riemann surface.

In this section, we'll show that the combinatorial Hodge-star operator on a triangulated surface induces similar structures. In particular, given any hermitian inner product on the complex valued simplicial 1-cochains, the harmonic cochains split as holomorphic and anti-holomorphic 1-cochains. We'll prove analogues of the bilinear relations of Riemann, and show how one obtains a combinatorial period matrix. This construction yields its own combinatorial Schottky-type problem, but we won't discuss that here.

After describing our combinatorial construction, we'll show that if the complex valued simplicial cochains of a triangulated closed orientable Riemannian 2manifold are equipped with the inner product induced by the Whitney embedding, then all of these structures provide a good approximation to their continuum analogues. In particular, the holomorphic and anti-holomorphic 1-cochains converge to the holomorphic and anti-holomorphic 1-forms, and the combinatorial period matrix converges to the conformal period matrix of the associated Riemann surface, as the mesh of the triangulation tends to zero. Hence, every conformal period matrix is a limit point of a sequence of combinatorial period matrices.

These statements may be interpreted as saying that a triangulation of a surface, endowed with an inner product on the associated cochains, determines a conformal structure. Furthermore, for triangulations of a Riemannian 2-manifold, a conformal structure is recovered (in the limit) from algebraic and combinatorial data. Statements like this have been expressed by physicists for some time in various field theories and in statistical mechanics; see [8].

We now describe the construction of combinatorial period matrices. First, we need to extend some of our definitions from previous sections to the case of complex valued cochains. Let $\langle$,$\rangle be any positive definite hermitian inner product on the$ complex valued simplicial 1-cochains of a triangulated topological surface $K$. We define the associated combinatorial star operator $\star$ by

$$
\langle\star a, b\rangle=(a \cup \bar{b})[M]
$$

where the bar denotes complex conjugation and $\cup$ is as in section 4 , extended over $\mathbb{C}$ linearly. Just as with real coefficients, we have a Hodge decomposition

$$
C^{1}(K) \cong \delta C^{0}(K) \oplus H^{1}(K) \oplus \delta^{*} C^{2}(K)
$$

where $H^{1}$ is the space of complex valued harmonic 1-cochains. By Lemma 5.2, $\star$ induces an isomorphism of $H^{1}$ and is skew-adjoint. Therefore, this map admits a unique polar decomposition $\star=H U$ where $H$ is symmetric positive definite and $U$ is unitary. Since $\star$ is skew-adjoint, so is $U$, and therefore the eigenvalues of $U$ are $\pm i$.

Definition 6.1. Let $K,\langle$,$\rangle , and \star$ be as above. Let $\star$ denote the map on harmonic cochains, as in Lemma 5.2, with polar decomposition $\star=H U$. We 


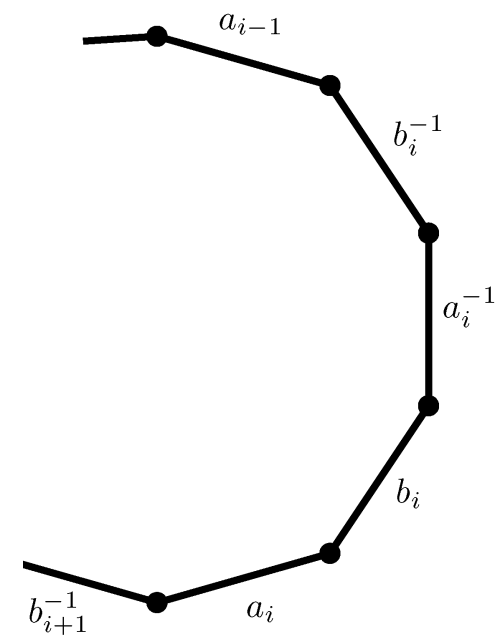

FiguRE 2. Fundamental domain of a surface

define the subspace of holomorphic 1-cochains by

$$
\mathcal{H}^{1,0}(K)=\left\{\sigma \in H^{1}(K) \mid U \sigma=-i \sigma\right\}
$$

and the subspace of anti-holomorphic 1-cochains by

$$
\mathcal{H}^{0,1}(K)=\left\{\sigma \in H^{1}(K) \mid U \sigma=i \sigma\right\} .
$$

Remark 6.2. An equivalent definition is to let $\mathcal{H}^{1,0}(K)$ be the span of the eigenvectors for non-positive imaginary eigenvalues of $\star$, and let $\mathcal{H}^{0,1}(K)$ be the span of the eigenvectors for non-negative imaginary eigenvalues of $\star$. This is clear from the polar decomposition of $\star$.

Lemma 6.3. Let $K$ be a triangulation of a surface $M$ of genus $g$. A hermitian inner product on the simplicial 1-cochains of $K$ gives an orthogonal direct sum decomposition

$$
H^{1}(K) \cong \mathcal{H}^{1,0} \oplus \mathcal{H}^{0,1}
$$

where $\mathcal{H}^{1,0}$ and $\mathcal{H}^{0,1}$ are defined as a above. Each summand on the right has complex dimension $g$ and complex conjugation maps $\mathcal{H}^{1,0}$ to $\mathcal{H}^{0,1}$ and vice versa.

Proof. The last assertion follows since $\star$ is $\mathbb{C}$-linear. The decomposition follows from properties of skew-adjoint operators: eigenspaces of distinct eigenvalues are orthogonal, and eigenvalues occur in conjugate pairs.

We now describe properties of the periods of the holomorphic and anti-holomorphic 1-cochains. We begin with a brief description of the homology basis we'll use to evaluate these cochain periods.

Without loss of generality, we assume that $M$ is obtained by identifying the sides of a $4 g$-gon, as in Figure 2. The basis $\left\{a_{1}, a_{2}, \ldots, a_{g}, b_{1}, b_{2}, \ldots, b_{g}\right\}$ for the first homology is classically referred to as the canonical basis [5], [15], since it satisfies the following nice property: the intersection of any two basis elements is non-zero only for $a_{j}$ and $b_{j}$, in which case it equals one. We assume our triangulation $K$ is a subdivision of the cellular decomposition given by the canonical homology basis. For any such subdivision, each element of the canonical homology basis is represented 


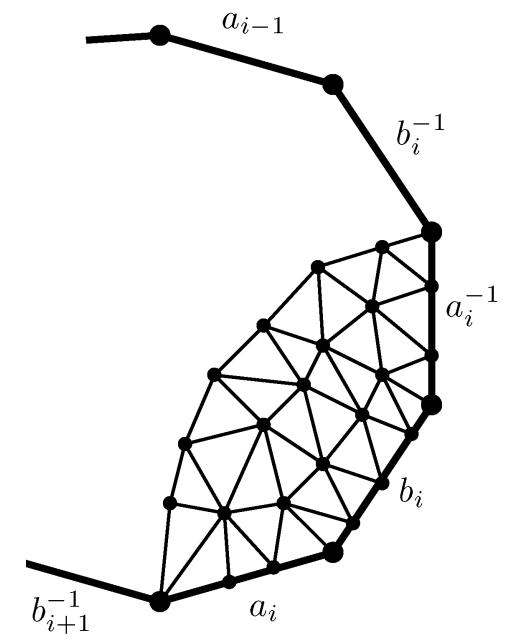

FiguRE 3. Triangulated surface

as a sum of the edges into which it is subdivided, as in Figure 3, Evaluating a cochain of $K$ on an element of the canonical homology basis means evaluating it on this subdivided representative.

Definition 6.4. For $h \in \mathcal{H}$, the $A$-periods and $B$-periods of $h$ are the following complex numbers:

$$
A_{j}=h\left(a_{j}\right), \quad B_{j}=h\left(b_{j}\right) \quad \text { for } \quad 1 \leq j \leq g .
$$

Theorem 6.5 (Riemann's bi-linear relations). If $\sigma, \sigma^{\prime} \in \mathcal{H}^{1,0}$ have $A$-periods $A_{j}$, $A_{j}^{\prime}$ and B-periods $B_{j}, B_{j}^{\prime}$ respectively, then

$$
\sum_{j=1}^{g}\left(A_{j} B_{j}^{\prime}-B_{j} A_{j}^{\prime}\right)=0 .
$$

Proof. The proof is an adaptation to the combinatorial setting of the elegant proof of the corresponding statement in the smooth setting; see for instance [15]. Let $\phi_{1}, \phi_{2}, \ldots, \phi_{g}$ be an orthonormal eigenbasis of $\mathcal{H}^{1,0}$ for $\star$, with eigenvalues $-i \lambda_{1},-i \lambda_{2}, \ldots,-i \lambda_{g}, \lambda_{j}>0$. Since $\phi_{j} \in \mathcal{H}^{1,0}, \overline{\sigma^{\prime}} \in \mathcal{H}^{0,1}$ and it follows that if $\sigma=\sum_{j=1}^{g} c_{j} \phi_{j}$, then

$$
\left\langle\star \sigma, \overline{\sigma^{\prime}}\right\rangle=-i \sum_{j=1}^{g} c_{j} \lambda_{j}\left\langle\phi_{j}, \overline{\sigma^{\prime}}\right\rangle=0 .
$$

The last equality is by Lemma 6.3. To show the bi-linear relation we compute

$$
\left\langle\star \sigma, \overline{\sigma^{\prime}}\right\rangle=\left(\sigma \cup \sigma^{\prime}\right)[M]
$$

where the fundamental class $[M]$ of $M$ may be represented by the sum of the 2cells of $K$ appropriately oriented. Now let $p: U \rightarrow M$ be the universal cover, with $U$ triangulated so that $p$ is locally a linear isomorphism onto the triangulation $K$ of $M$. Let $S$ denote a fundamental domain in the triangulation of $U$ so that the 


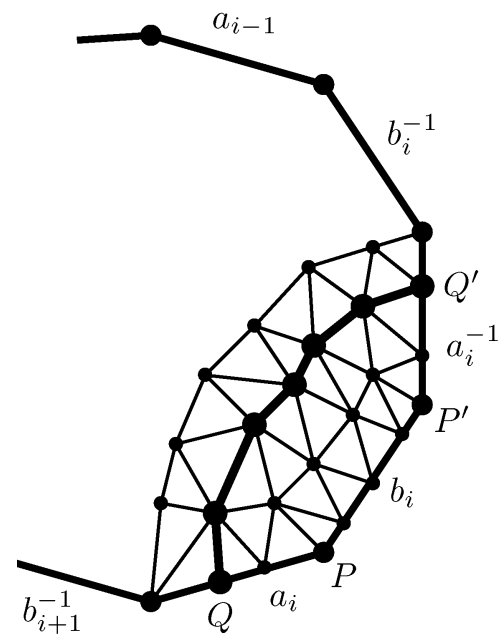

Figure 4. A 1-cycle $\alpha$ from $Q$ to $Q^{\prime}$

induced map $p_{*}$ maps the 2-simplices of $S$ isomorphically onto the 2-simplices of $K$. Then $p_{*}(S)=[M]$, so the last expression equals

$$
\left(\sigma \cup \sigma^{\prime}\right)[M]=\left(p^{*} \sigma \cup p^{*} \sigma^{\prime}\right)(S)
$$

where $p^{*}$ denotes the pull back on cohomology. Since $\sigma$ is holomorphic, it is closed, as is $p^{*} \sigma$. Since $\bar{S}$ is contractible to a point, the restriction of $p^{*} \sigma$ to $\bar{S}$ may be written as $p^{*} \sigma=\delta f$ for some 0 -cochain $f$. Thus, since $\delta \sigma^{\prime}=0$ we have

$$
\begin{aligned}
\left(p^{*} \sigma \cup p^{*} \sigma^{\prime}\right)(S) & =\left(\delta f \cup p^{*} \sigma^{\prime}\right)(S) \\
& =\left(f \cup p^{*} \sigma^{\prime}\right)(\partial S) \\
& =\sum_{j=1}^{g}\left(f \cup p^{*} \sigma^{\prime}\right)\left(a_{j}+a_{j}^{-1}+b_{j}+b_{j}^{-1}\right) .
\end{aligned}
$$

It remains to show that this last expression equals $\sum_{j=1}^{g}\left(A_{j} B_{j}^{\prime}-B_{j} A_{j}^{\prime}\right)$. To do this, we first derive a simple relation for the values of $f$ on the 0 -simplices contained in the cycles of the canonical homology basis. Consider Figure 4. The chain $\alpha$ from $Q$ to $Q^{\prime}$ is a cycle. Since $\alpha$ is homologous to the cycle made up of chains from $Q$ to $P, P$ to $P^{\prime}$ and $P^{\prime}$ to $Q^{\prime}$, and since the first and third project to the same chains on $K$, we have that

$$
f(Q)-f\left(Q^{\prime}\right)=f(\partial \alpha)=\delta f(\alpha)=p^{*} \sigma(\alpha)=p^{*} \sigma\left(b_{j}\right)=B_{j}
$$

which means that for any 1-cochain $\tau$

$$
(f \cup \tau)\left(a_{j}^{-1}\right)=-\left(\left(f+B_{j}\right) \cup \tau\right)\left(a_{j}\right)=-(f \cup \tau)\left(a_{j}\right)-B_{j} \tau\left(a_{j}\right) .
$$

Similarly,

$$
(f \cup \tau)\left(b_{j}^{-1}\right)=-\left(\left(f-A_{j}\right) \cup \tau\right)\left(b_{j}\right)=-(f \cup \tau)\left(b_{j}\right)+A_{j} \tau\left(a_{j}\right) .
$$




\begin{tabular}{c|cccccccc} 
& $a_{1}$ & $a_{2}$ & $\cdots$ & $a_{g}$ & $b_{1}$ & $b_{2}$ & $\cdots$ & $b_{g}$ \\
\hline$\sigma_{1}$ & 1 & 0 & $\cdots$ & 0 & $\sigma_{1}\left(b_{1}\right)$ & $\sigma_{1}\left(b_{2}\right)$ & $\cdots$ & $\sigma_{1}\left(b_{g}\right)$ \\
$\sigma_{2}$ & 0 & 1 & $\cdots$ & 0 & $\sigma_{2}\left(b_{1}\right)$ & $\sigma_{2}\left(b_{2}\right)$ & $\cdots$ & $\sigma_{2}\left(b_{g}\right)$ \\
$\vdots$ & & & $\ddots$ & & & & & \\
$\sigma_{g}$ & 0 & 0 & $\cdots$ & 1 & $\sigma_{g}\left(b_{1}\right)$ & $\sigma_{g}\left(b_{2}\right)$ & $\cdots$ & $\sigma_{g}\left(b_{g}\right)$
\end{tabular}

Figure 5. The period matrix

So, we finally have that

$$
\begin{aligned}
0=\left\langle\star \sigma, \overline{\sigma^{\prime}}\right\rangle & =\sum_{j=1}^{g}\left(f \cup p^{*} \sigma^{\prime}\right)\left(a_{j}+a_{j}^{-1}+b_{j}+b_{j}^{-1}\right) \\
& =\sum_{j=1}^{g}-B_{j} p^{*} \sigma^{\prime}\left(a_{j}\right)+A_{j} p^{*} \sigma^{\prime}\left(b_{j}\right) \\
& =\sum_{j=1}^{g}\left(A_{j} B_{j}^{\prime}-B_{j} A_{j}^{\prime}\right) .
\end{aligned}
$$

Retracing the proof and replacing $\overline{\sigma^{\prime}}$ with $\sigma$, we see that if $\sigma=\sum_{j=1}^{g} c_{j} \phi_{j}$ is holomorphic, then

$$
\sum_{j=1}^{g} \lambda_{j}\left|c_{j}\right|^{2}=i\langle\star \sigma, \sigma\rangle=i \sum_{j=1}^{g}\left(A_{j} \overline{B_{j}}-B_{j} \overline{A_{j}}\right) \geq 0 .
$$

This expression yields

Corollary 6.6. Let $\sigma$ be a holomorphic 1-cochain.

(1) If the A-periods or B-periods of $\sigma$ vanish, then $\sigma=0$.

(2) If the A-periods and B-periods of $\sigma$ are real, then $\sigma=0$.

Proof. Either condition in the statement implies the right hand side of equation (11) is zero. Since $\lambda_{j}>0$ for all $j, c_{j}=0$ for all $j$, so $\sigma=0$.

Now let $\left\{\tau_{1}, \tau_{2}, \ldots, \tau_{g}\right\}$ be any basis for the space of holomorphic cochains. As just proved, if all the $A$-periods of a linear combination of these basis elements vanish, then this linear combination is identically zero. This implies that we can solve uniquely for coefficients $c_{i, j}$ such that

$$
\sum_{i=1}^{g} c_{i, j} \tau_{i}\left(a_{k}\right)=\delta_{j, k}
$$

We put $\sigma_{j}=\sum_{i=1}^{g} c_{i, j} \tau_{i}$ and we call the basis $\left\{\sigma_{1}, \sigma_{2}, \ldots, \sigma_{g}\right\}$ the canonical basis for the space of holomorphic 1-cochains. This gives a matrix of periods as in Figure 5 .

Definition 6.7. Let $\left\{\sigma_{1}, \sigma_{2}, \ldots, \sigma_{g}\right\}$ be the canonical basis for the space of holomorphic 1-cochains and $\left\{a_{1}, a_{2}, \ldots, a_{g}, b_{1}, b_{2}, \ldots, b_{g}\right\}$ the canonical homology basis, 
so $\sigma_{i}\left(a_{j}\right)=\delta_{i, j}$. We define the period matrix $\Pi=\left(\pi_{i . j}\right)$ to be the $g \times g$ matrix of $B$-periods:

$$
\pi_{i, j}=\sigma_{i}\left(b_{j}\right) .
$$

When we wish to emphasize the dependence of $\Pi$ on $K$, we'll write $\Pi_{K}$.

Remark 6.8. Let $K$ be fixed. If two inner products on $C^{1}(K)$ differ by a constant multiple, then the associated period matrices are equal. Hence, the combinatorial period matrix is a "conformal invariant".

Theorem 6.9. Let $K$ be a triangulated closed surface with a simplicial cochain inner product. The associated period matrix $\Pi$ is symmetric and $\operatorname{Im}(\Pi)$ is positive definite.

Proof. It suffices to show $\sigma_{i}\left(b_{j}\right)=\sigma_{j}\left(b_{i}\right)$ for $1 \leq i, j \leq g$, where $\sigma_{i}$ and $\sigma_{j}$ are canonical holomorphic cochain basis elements. We apply Theorem 6.5 and compute:

$$
\begin{aligned}
0 & =\sum_{k=1}^{g} \sigma_{i}\left(a_{k}\right) \sigma_{j}\left(b_{k}\right)-\sigma_{i}\left(b_{k}\right) \sigma_{j}\left(a_{k}\right) \\
& =\sum_{k=1}^{g} \delta_{i, k} \sigma_{j}\left(b_{k}\right)-\delta_{j, k} \sigma_{i}\left(b_{k}\right) \\
& =\sigma_{j}\left(b_{i}\right)-\sigma_{i}\left(b_{j}\right)
\end{aligned}
$$

To prove the second statement, let $\sigma=\sum_{i=1}^{g} c_{i} \sigma_{i}$ be a non-trivial $\mathbb{R}$-linear combination of elements of the canonical basis of holomorphic cochains. Then $\sigma\left(a_{i}\right)=c_{i}$. We show

$$
\sigma \cdot \operatorname{Im}(\Pi) \cdot \sigma>0
$$

by using equation (11) and computing

$$
\begin{aligned}
0 & <i \sum_{k=1}^{g} \sigma\left(a_{k}\right) \overline{\sigma\left(b_{k}\right)}-\sigma\left(b_{k}\right) \overline{\sigma\left(a_{k}\right)} \\
& =i \sum_{k=1}^{g} c_{k}\left(\sum_{i=1}^{g} c_{i} \overline{\sigma_{i}\left(b_{k}\right)}\right)-c_{k}\left(\sum_{i=1}^{g} c_{i} \sigma_{i}\left(b_{k}\right)\right) \\
& =i \sum_{i=1}^{g} \sum_{k=1}^{g} c_{k} c_{i} \overline{\sigma_{i}\left(b_{k}\right)}-c_{k} c_{i} \sigma_{i}\left(b_{k}\right) \\
& =2 \sum_{i=1}^{g} \sum_{k=1}^{g} c_{k} c_{i} \operatorname{Im}\left(\sigma_{i}\left(b_{k}\right)\right) \\
& =2(\sigma \cdot \operatorname{Im}(\Pi) \cdot \sigma) .
\end{aligned}
$$

Up to this point, we have assumed that $K$ is a triangulated closed topological surface and $\langle$,$\rangle is a positive definite inner product on the simplicial cochains of$ $K$. As remarked at the beginning of this section, the structures we have uncovered (splitting of harmonics, bilinear relations, period matrix, etc.) also appear for 1forms on a Riemann surface. In fact, all of the statements proven above hold for forms as well [15]. 


\section{Convergence OF PERIOD MATRICES}

Let $M$ be an orientable closed Riemannian 2-manifold. The Riemannian metric induces an operator $\star$ which squares to $-\mathrm{Id}$, and (identifying tangent and cotangent space via the metric) this operator $\star$ gives an almost complex structure. Gauss proved that $M$ admits a unique complex structure, i.e. a Riemann surface structure, that is compatible with this almost complex structure. This theorem is, a priori, non-trivial, and involves a transcendental construction of holomorphic coordinate charts. By Torelli's theorem the resulting complex structure is determined uniquely by the period matrix of the associated Riemann surface $M$. We now describe how this conformal period matrix of $M$ is related to a combinatorial period matrix in the case that $M$ is triangulated.

Let $K$ be a triangulation of a Riemannian 2-manifold $M$. The inner product on the vector space of real valued 1 -forms may be extended to a hermitian inner product on the space of complexified 1-forms canonically, by declaring

$$
\left\langle\omega_{1} \otimes z_{1}, \omega_{2} \otimes z_{2}\right\rangle=z_{1} \overline{z_{2}}\left\langle\omega_{1}, \omega_{2}\right\rangle
$$

for $\omega_{i} \otimes z_{i} \in T^{*} M \otimes \mathbb{C}$. Let \|\| denote the induced norm on $T^{*} M \otimes \mathbb{C}$.

The Whitney embedding of complex valued 1-cochains into $T^{*} M \otimes \mathbb{C}$ induces an inner product on complex valued 1-cochains. For the remainder of this section, we work only with this inner product. We remark here that while the approximation theorems from sections 3 and 5 (using the Whitney inner product) involved realvalued forms and cochains, the proofs hold verbatim for complex coefficients as well.

First we prove the following

Lemma 7.1. Let $M$ be a Riemannian 2-manifold with triangulation $K$ of mesh $\eta$, and let $\mathfrak{h}$ be a complex valued holomorphic 1-form on $M$, so $\star \mathfrak{h}=-i \mathfrak{h}$. By the Hodge decomposition of cochains and Lemma 6.3 we may write

$$
R \mathfrak{h}=\delta g+h_{1}+h_{2}+\delta^{*} k
$$

uniquely for $h_{1} \in \mathcal{H}^{1,0}$ and $h_{2} \in \mathcal{H}^{0,1}$. Then there exists a positive constant $C$, dependent on $\mathfrak{h}$ but independent of $K$, such that

$$
\left\|W h_{1}-\mathfrak{h}\right\| \leq C \cdot \eta
$$

Proof. By Theorems 3.10 and 5.5 there is a constant $C$, independent of $K$, such that

$$
\begin{aligned}
C \cdot \eta & \geq\left\|W \star\left(h_{1}+h_{2}\right)-\star \mathfrak{h}\right\|+\left\|\mathfrak{h}-W\left(h_{1}+h_{2}\right)\right\| \\
& =\left\|W \star\left(h_{1}+h_{2}\right)-\star \mathfrak{h}\right\|+\left\|\star \mathfrak{h}+i W\left(h_{1}+h_{2}\right)\right\| \\
& \geq\left\|W \star h_{1}+W \star h_{2}+i W\left(h_{1}+h_{2}\right)\right\| \\
& =\left\|\star h_{1}+\star h_{2}+i\left(h_{1}+h_{2}\right)\right\| .
\end{aligned}
$$

Let $\phi_{1}, \phi_{2}, \ldots, \phi_{g}$ be an orthonormal eigenbasis of $\mathcal{H}^{1,0}$ for $\star$, with eigenvalues $-i \lambda_{1},-i \lambda_{2}, \ldots,-i \lambda_{g}, \lambda_{j}>0$, and let $\phi_{1}^{\prime}, \phi_{2}^{\prime}, \ldots, \phi_{g}^{\prime}$ be an orthonormal eigenbasis of $\mathcal{H}^{0,1}$ for $\star$, with eigenvalues $i \lambda_{1}^{\prime}, i \lambda_{2}^{\prime}, \ldots, i \lambda_{g}^{\prime}, \lambda_{j}^{\prime}>0$. Since $h_{1} \in \mathcal{H}^{1,0}$ and $h_{2} \in \mathcal{H}^{0,1}$ we may write $h_{1}=\sum_{j=1}^{g} c_{j} \phi_{j}$ and $h_{2}=\sum_{j=1}^{g} c_{j}^{\prime} \phi_{j}^{\prime}$. Then using the fact 
that $\mathcal{H}^{1,0} \perp \mathcal{H}^{0,1}$ we have

$$
\begin{aligned}
C^{2} \cdot \eta^{2} & \geq\left\|\sum_{j=1}^{g}\left(1-\lambda_{j}\right) c_{j} \phi_{j}\right\|^{2}+\left\|\sum_{j=1}^{g}\left(1+\lambda_{j}^{\prime}\right) c_{j}^{\prime} \phi_{j}^{\prime}\right\|^{2} \\
& =\sum_{j=1}^{g}\left(1-\lambda_{j}\right)^{2}\left|c_{j}\right|^{2}+\sum_{j=1}^{g}\left(1+\lambda_{j}^{\prime}\right)^{2}\left|c_{j}^{\prime}\right|^{2} \\
& \geq \sum_{j=1}^{g}\left|c_{j}^{\prime}\right|^{2} \\
& =\left\|h_{2}\right\|^{2} .
\end{aligned}
$$

So we conclude

$$
\left\|W h_{1}-\mathfrak{h}\right\| \leq\left\|W\left(h_{1}+h_{2}\right)-\mathfrak{h}\right\|+\left\|h_{2}\right\| \leq 2 C \cdot \eta .
$$

One can check that the hermitian inner product on 1-forms of $M$, defined in (2), agrees with the usual inner product on the 1-forms of the Riemann surface associated to $M$, given by

$$
\langle\omega, \eta\rangle=\int_{M} \omega \wedge \star \bar{\eta}
$$

It is a peculiarity of working in the middle dimension (here 1) that this inner product, and the Hodge star operator, depend only on the conformal class of the Riemannian metric. This implies that the period matrix of the Riemann surface associated to $M$ can be computed by using the inner product in (2) in the following way: split off the harmonic 1-forms and evaluate the appropriate basis of the $-i$ eigenspace of $\star$ on the canonical homology basis. Note that this involves a transcendental procedure in the Hodge decomposition of forms. The point of the following theorem is that the period matrix, and therefore by Torelli's theorem the complex structure, is computable, to any desired accuracy, from finite algebraic and combinatorial data.

Theorem 7.2. Let $M$ be a closed orientable Riemannian 2-manifold and let $\Pi$ be the period matrix of the Riemann surface associated to $M$. Let $K_{n}$ be a sequence of triangulations of $M$ with mesh converging to zero. Then, for each $n$, the induced Whitney inner product on the simplicial 1-cochains of $K_{n}$ gives rise to a combinatorial period matrix $\Pi_{K_{n}}$, and

$$
\lim _{n \rightarrow \infty} \Pi_{K_{n}}=\Pi \text {. }
$$

Proof. Let $\mathfrak{h}_{g}, \ldots, \mathfrak{h}_{g}$ be the canonical basis of holomorphic 1-forms with periods

$$
\begin{aligned}
& \mathfrak{h}_{i}\left(a_{j}\right)=\int_{a_{j}} \mathfrak{h}_{i}=\delta_{i, j}, \\
& \mathfrak{h}_{i}\left(b_{j}\right)=\int_{b_{j}} \mathfrak{h}_{i}=\pi_{i, j}
\end{aligned}
$$

for $1 \leq i, j \leq g$, and $\pi_{i, j}$ the $(i, j)$ entry of $\Pi$. 
For each $n$, let $\varphi_{1}^{n}, \ldots, \varphi_{g}^{n}$ be the canonical basis for the holomorphic cochains of $K_{n}$. Then the periods are

$$
\begin{aligned}
\varphi_{i}^{n}\left(a_{j}\right) & =\delta_{i, j}, \\
\varphi_{i}^{n}\left(b_{j}\right) & =\pi_{i, j}^{n}
\end{aligned}
$$

for $1 \leq i, j \leq g$, and $\pi_{i, j}^{n}$ the $(i, j)$ entry of $\Pi_{K_{n}}$. Our goal is to show, for all $1 \leq i, j \leq g$,

$$
\lim _{n \rightarrow \infty} \varphi_{i}^{n}\left(b_{j}\right)=\mathfrak{h}_{i}\left(b_{j}\right)
$$

Let $R_{n}$ denote the integration map taking 1 -forms to cochains on $K_{n}$. We denote by $h_{i}^{n}$ the holomorphic part of the cochain $R_{n} \mathfrak{h}_{i}$. By the previous lemma, $h_{i}^{n} \rightarrow \mathfrak{h}_{i}$ as $n \rightarrow \infty$. Hence,

$$
\lim _{n \rightarrow \infty} h_{i}^{n}\left(a_{j}\right)=\mathfrak{h}_{i}\left(a_{j}\right)=\delta_{i, j}
$$

For each $n$ and $1 \leq i \leq g$ we may write

$$
h_{i}^{n}=\sum_{k=1}^{g} c_{i, k}^{n} \varphi_{k}^{n}
$$

and by evaluating on the cycle $a_{j}$ we see that

$$
c_{i, j}^{n}=\sum_{k=1}^{g} c_{i, k}^{n} \varphi_{k}^{n}\left(a_{j}\right)=h_{i}^{n}\left(a_{j}\right) .
$$

Combining this with equation (3), we have

$$
\lim _{n \rightarrow \infty} c_{i, j}^{n}=\delta_{i, j}
$$

which implies

$$
\lim _{n \rightarrow \infty}\left\|\varphi_{i}^{n}-h_{i}^{n}\right\|=0
$$

By the lemma, $\left\|h_{i}^{n}\right\| \rightarrow\left\|\mathfrak{h}_{i}\right\|$, so the sequences $\left\|h_{i}^{n}\right\|$ and $\left\|\varphi_{i}^{n}\right\|$ are bounded. Finally, we have

$$
\lim _{n \rightarrow \infty} \varphi_{i}^{n}\left(b_{j}\right)=\lim _{n \rightarrow \infty} h_{i}^{n}\left(b_{j}\right)=\mathfrak{h}_{i}\left(b_{j}\right)
$$

Corollary 7.3. Let $M$ be a closed Riemann surface with period matrix $\Pi$. Let $K_{n}$ be a sequence of triangulations of $M$ with mesh converging to zero, and combinatorial period matrices $\Pi_{K_{n}}$ induced by the Whitney metric. Then,

$$
\lim _{n \rightarrow \infty} \Pi_{K_{n}}=\Pi \text {. }
$$

Proof. While there isn't a notion of geodesic length on a Riemann surface, a 'distance converging to zero' is well defined since it depends only on a conformal class of metrics. So the statement of the corollary makes sense. Then one can choose any Riemannian metric on $M$ in the conformal class of metrics determined by the conformal structure of $M$, and apply the above theorem.

The following corollary is related to the Schottky problem; it gives necessary conditions for a matrix to be the period matrix of some Riemann surface:

Corollary 7.4. Every conformal period matrix is the limit of a sequence of combinatorial period matrices. 


\section{REFERENCES}

[1] Dodziuk, J. "Finite-Difference Approach to the Hodge Theory of Harmonic Forms," Amer. J. of Math. 98 No. 1 (1976) 79-104. MR0407872 (53:11642)

[2] Dodziuk J. and Patodi V. K. "Riemannian Structures and Triangulations of Manifolds," Journal of Indian Math. Soc. 40 (1976) 1-52. MR0488179 (58:7742)

[3] Dupont, J. "Curvature and Characteristic Classes," Lecture Notes in Mathematics, vol. 640, Springer-Verlag (1978). MR0500997 (58:18477)

[4] Eckmann, B. "Harmonische Funktionnen und Randvertanfgaben in einem Komplex," Commentarii Math. Helvetici, 17 (1944-45), 240-245. MR0013318 (7:138f)

[5] Farkas, H. and Kra, I. "Riemann Surfaces," Springer-Verlag (1991). MR1139765 (93a:30047)

[6] Gu, X. and Yau, S.T. "Computing Conformal Structures of Surfaces," Comm. Inf. Sys. 2 No. 2 (2002) 121-146. MR1958012 (2003m:65023)

[7] Manin, Y. "The Partition Function of the Polyakov String can be Expressed in Terms of Theta-Functions," Phys. Lett. B 172 No. 2 (1986) 184-185. MR.844733 (87k:81120)

[8] Costa-Santos, R. and McCoy, B.M. "Finite Size Corrections for the Ising Model on Higher Genus Triangular Lattices," J. Statist. Phys. 112 Nos. 5-6 (2003) 889-920. MR2000227 (2004h:82021)

[9] Mercat, C. "Discrete Riemann Surfaces and the Ising Model," Comm. Math. Phys. 218 (2001), No. 1, 177-216. MR.1824204 (2002c:82019)

[10] Mercat, C. "Discrete period Matrices and Related Topics," arxiv.org math-ph/0111043, June 2002.

[11] Mercat, C. "Discrete Polynomials and Discrete Holomorphic Approximation," arxiv.org math-ph/0206041.

[12] Ranicki, A. and Sullivan, D. "A Semi-local Combinatorial Formula for the Signature of a 4k-manifold," J. Diff. Geometry, Vol. II (1976), 23-29. MR0423366 (54:11345)

[13] Smits, L. "Combinatorial Approximation to the Divergence of 1-forms on Surfaces," Israel J. of Math. 75 (1991) 257-271. MR.1164593 (93d:57052)

[14] Spivak, M. A. "Comprehensive Introduction to Differential Geometry," Vol. IV, Publish or Perish Inc., Boston, MA, 1975. MR0394452 (52:15254a)

[15] Springer, G. "Introduction to Riemann Surfaces," Addison-Wesley Publ. Company (1957). MR0092855 (19:1169g)

[16] Whitney, H. "Geometric Integration Theory," Princeton Univ. Press (1957). MR0087148 $(19: 309 \mathrm{c})$

[17] Whitney, H. "On Products in a Complex," Annals of Math. (2) 39 (1938) 397-432. MR.1503416

[18] Wilson, S.O. "Cochain Algebra on Manifolds and Convergence Under Refinement," Topology and Its Applications 154 No. 9 (2007) 1898-1920. MR.2319262

School of Mathematics, University of Minnesota-Twin Cities, 127 Vincent Hall, 206 Church St. S.E., Minneapolis, Minnesota 55455

E-mail address: scottw@math.umn.edu 